\title{
Determination of solid phase chemical diffusion coefficient and density of states by electrochemical methods: Application to iridium oxide-based thin films
}

\author{
Jonas Backholm, ${ }^{1, a)}$ Peter Georén, ${ }^{2}$ and Gunnar A. Niklasson ${ }^{1}$ \\ ${ }^{1}$ Department of Engineering Sciences, The Angström Laboratory, Uppsala University, \\ P.O. Box 534, SE-75121 Uppsala, Sweden \\ ${ }^{2}$ ChromoGenics Sweden AB, Märstagatan 4, SE-75323 Uppsala, Sweden
}

(Received 20 September 2007; accepted 13 November 2007; published online 17 January 2008)

\begin{abstract}
Potentiostatic intermittent titration technique (PITT) and electrochemical impedance spectroscopy (EIS) were investigated as methods to determine solid phase chemical diffusion coefficient $(D)$ and electronic density of states (DOS). These techniques were then applied to iridium oxide (IrOx) and iridium-tantalum oxide (IrTaOx) thin films prepared by sputter deposition. The experiments, performed in $1 M$ propionic acid between -0.2 and $0.8 \mathrm{~V} \mathrm{vs} \mathrm{Ag} / \mathrm{AgCl}$, showed effects of interfacial side reactions, whose contribution to the electrochemical response could be identified and corrected for in the case of PITT as well as EIS. It was found that $D$ is strongly underestimated when using PITT with the common Cottrell formalism, which follows from non-negligible interfacial charge transfer and Ohmic resistances. EIS indicated an anomalous diffusion mechanism, and $D$ was determined to be in the $10^{-11}-10^{-10} \mathrm{~cm}^{2} / \mathrm{s}$ range for IrOx and IrTaOx. Both PITT and EIS showed that the intercalated charge as a function of potential exhibits a shape that resembles the theoretical DOS of crystalline iridium oxide, especially for IrTaOx. (C) 2008 American Institute of Physics. [DOI: $10.1063 / 1.2831484]$
\end{abstract}

\section{INTRODUCTION}

Electrochemical methods are widely used to investigate ion transport and diffusion in liquid electrolytes ${ }^{1}$ as well as in solids. ${ }^{2}$ Systems with mixed ionic and electronic conduction are of importance for a number of applications, such as batteries, ${ }^{3}$ electrochemical sensors, ${ }^{4}$ and electrochromic (EC) devices. ${ }^{5}$ The coupled ion-electron transport is in general very complex, ${ }^{6,7}$ but considerable simplification is possible in the case of chemical diffusion of ions intercalated into a material. The coupled ion-electron transport is then described by the chemical diffusion coefficient $(D)$. Galvanostatic intermittent titration technique (GITT), potentiostatic intermittent titration technique (PITT), and electrochemical impedance spectroscopy (EIS) are frequently used methods to determine $D$ of guest species in various inorganic hosts. ${ }^{8-10}$ Recent advances regarding the analysis of these methods include correction for non-negligible charge transfer and Ohmic resistances in the PITT case ${ }^{11}$ and introduction of anomalous diffusion when analyzing EIS data. ${ }^{12}$

In chronopotentiometry (CP) and GITT experiments the quasi-steady-state potential is measured as ions are intercalated into the material. Recently, it has become apparent that the variation of the potential can often be related to the electronic density of states (DOS) of the host material. ${ }^{13}$ Hence, the GITT and CP methods have been used to study the electronic DOS in the vicinity of the Fermi level for several transition metal oxides. ${ }^{14,15}$ There are other methods to determine the DOS experimentally, especially electron and $\mathrm{x}$-ray spectroscopic methods, ${ }^{16-18}$ and theoretical ab initio

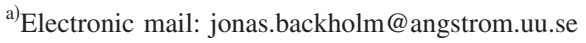

calculations can be used too. ${ }^{19-22}$ A detailed knowledge of the DOS is especially interesting for EC materials, since the coloration in the visible wavelength region involves electron excitations between filled and empty states by, for example, an intervalence charge transfer mechanism. ${ }^{23}$

The accuracy of the methods for determining $D$ and the DOS may be severely limited in the presence of side reactions. These are not uncommon in electrochemical systems, but are often neglected or not reported in detail. The present paper investigates PITT and EIS, using the recent theoretical advances by Montella ${ }^{11}$ and Bisquert and Compte, ${ }^{12}$ as methods for the determination of the solid phase chemical diffusion coefficient and the DOS. In particular, we show that the side reactions can be corrected for explicitly. Specifically, we use the above methods to study two iridium based oxides that can be used in electrochromic devices as anodic counter electrodes to the cathodic tungsten oxide. ${ }^{24-28}$ Compared to nickel oxide, which is a more frequently used anodic electrochromic oxide, ${ }^{5,29}$ the iridium based oxides are less coloring but they show greater chemical stability. To improve the electrochromic behavior of iridium oxide, and to make it less expensive for applications, other metals, such as Sn or Ta, are added to obtain a mixed electrochromic oxide. ${ }^{30-32}$

\section{EXPERIMENT}

Samples of iridium oxide ( $\mathrm{IrOx}$ ) and iridium-tantalumoxide ( $\mathrm{IrTaOx}$ ) were prepared using reactive dc magnetron sputtering onto $\mathrm{In}_{2} \mathrm{O}_{3}: \mathrm{Sn}$ (ITO) covered glass substrates. The sheet resistance of the ITO was $10 \Omega$. A multitarget sputter system, based on a Balzer UTT400 unit, was used with 99.95\% pure metallic $5 \mathrm{~cm}$ diameter targets positioned $13 \mathrm{~cm}$ from the substrate. The deposition took place in a 
99.995\% pure argon-oxygen atmosphere at 30 mTorr. The $\mathrm{O}_{2}$ / Ar flow ratio was 1 when depositing $\mathrm{IrOx}$ films and 0.13 when depositing IrTaOx films. The sputtering power was $200 \mathrm{~W}$ during deposition of $\mathrm{IrOx}$ and $100 \mathrm{~W}$ for $\mathrm{Ir}$ and $220 \mathrm{~W}$ for Ta during deposition of IrTaOx. Typical deposition rates and film thicknesses were determined to be 1.4 and $0.8 \mathrm{~nm} / \mathrm{s}$ for $\operatorname{IrOx}(85 \mathrm{~nm})$ and $\operatorname{IrTaOx}(95 \mathrm{~nm})$, respectively, using an Alpha Step profilometer. Additional structural characterization by atomic force microscopy, x-ray diffraction, transmission electron microscopy (TEM), Rutherford backscattering spectroscopy, and x-ray photoelectron spectroscopy of both types of films were presented in two recent papers. ${ }^{32,33}$ The compositions of the as-deposited films were $\mathrm{IrO}_{2.2}$ and $\mathrm{IrTa}_{1.4} \mathrm{O}_{5.6}$, respectively, with a surface roughness of about $9 \mathrm{~nm}$ and a grain size of 3-4 nm for both types of films. Preliminary observations of cross section TEM images show that IrTaOx has a diffuse columnar structure, whereas the $\mathrm{IrOx}$ has a more porous structure, which is sensitive to electron beam irradiation.

All of the electrochemical measurements were performed using a standard three-electrode setup with the electrochromic film as working electrode, a $1 \mathrm{~cm}^{2}$ platinum counter electrode, and a $\mathrm{Ag} / \mathrm{AgCl}$ reference electrode submerged in $1 M$ propionic acid. Prior to the PITT and EIS studies, the films were pretreated using cyclic voltammetry $(\mathrm{CV})$ until a stable response was obtained ( $\sim 10$ cycles). The $\mathrm{CV}$ was performed between -0.3 and $+1 \mathrm{~V}$ versus the $\mathrm{Ag} / \mathrm{AgCl}$ reference electrode at $20 \mathrm{mV} / \mathrm{s}$ using an Ecochemie Autolab/GPES interface. One of our previous studies ${ }^{33}$ indicated a $10 \%$ increase of oxygen content in the films during these initial cycles.

During the PITT experiments, performed using the Ecochemie Autolab/GPES interface, the potential was first decreased using $-50 \mathrm{mV}$ potential steps from +1 to $-0.3 \mathrm{~V}$ to intercalate the films, and then increased back to $+1 \mathrm{~V}$ using $+50 \mathrm{mV}$ steps to deintercalate the films. The relaxation time between each step was set to $3600 \mathrm{~s}$ to ensure as relaxed films as possible prior to each step. During the experiment the Faradaic current was monitored during the first $300 \mathrm{~s}$.

Impedance spectroscopy was performed using a Solartron 1260 frequency response analyzer together with a Solartron 1286 electrochemical interface, enabling bias potential rejection for better accuracy. The spectra were recorded using a $10 \mathrm{mV}$ amplitude ac potential between $1 \mathrm{MHz}$ and $10 \mathrm{mHz}$ with 10 points per decade using an autointegration routine, giving noise to signal ratio less than 0.001 . Spectra were recorded successively at 0.8 to $-0.2 \mathrm{~V}$ and then back to $0.8 \mathrm{~V}$ vs $\mathrm{Ag} / \mathrm{AgCl}$ with $0.2 \mathrm{~V}$ intervals after applying the potential for $1800 \mathrm{~s}$, allowing the films to attain a steady state.

\section{RESULTS AND ANALYSIS: DIFFUSION PITT}

PITT has for a long time been used as a method to determine the chemical diffusion coefficient for intercalation materials. It has been established ${ }^{9}$ that the charge compen-
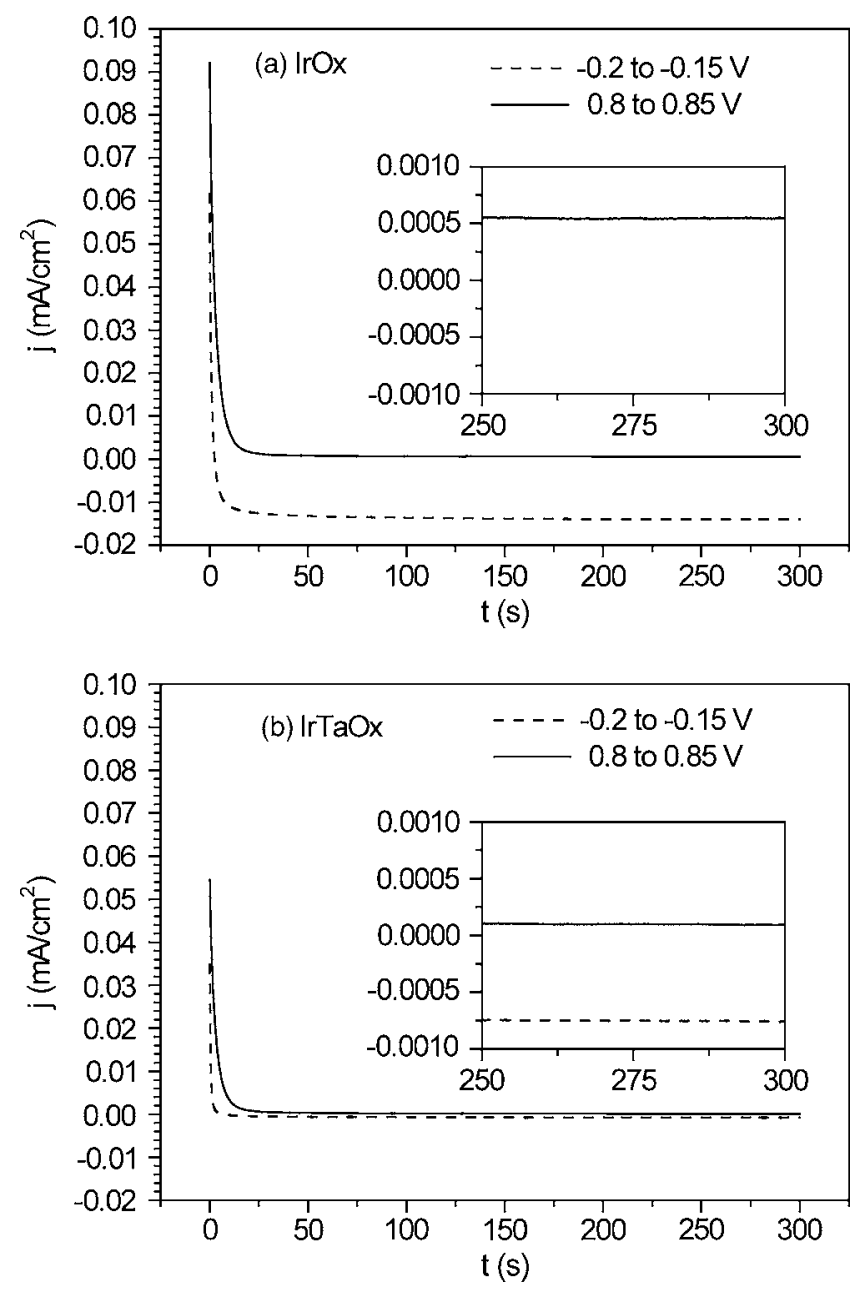

FIG. 1. Current response to $50 \mathrm{mV}$ potential steps, from -0.2 to $-0.15 \mathrm{~V}$ and 0.8 to $0.85 \mathrm{~V}$ vs $\mathrm{Ag} / \mathrm{AgCl}$, as a function of time for IrOx (a) and $\operatorname{IrTaOx}(\mathrm{b})$. The inset shows the current in detail between 250 and $300 \mathrm{~s}$, for the step at high potential in (a) and both steps in (b).

sating current during a PITT step can be derived from Fick's second law with the appropriate initial and boundary conditions. The current can be expressed as

$$
I(t)=\frac{\Delta Q D^{1 / 2}}{L \pi^{1 / 2}} \frac{1}{t^{1 / 2}} \quad \text { if } t \ll \frac{L^{2}}{D}
$$

or

$$
I(t)=\frac{2 \Delta Q D}{L^{2}} \exp \left(-\frac{\pi^{2} D}{4 L^{2}} t\right) \quad \text { if } t \gg \frac{L^{2}}{D},
$$

where $\Delta Q$ is the intercalated charge corresponding to the potential step, $D$ is the chemical diffusion coefficient, $L$ is the electrode thickness, and $t$ is the time. The chemical diffusion coefficient can thereby be determined by looking at the short time region where $I t^{1 / 2}$ is time independent, normally called the Cottrell region, or by looking at the long time region where the current decreases exponentially.

Figure 1 shows two typical current responses to potential steps for (a) $\mathrm{IrOx}$ and (b) IrTaOx, one at a high potential $(0.8 \mathrm{~V}$ vs $\mathrm{Ag} / \mathrm{AgCl})$ and the other at a low potential $(-0.2 \mathrm{~V}$ vs $\mathrm{Ag} / \mathrm{AgCl}$ ). It can be seen in Eq. (2) that the current is expected to approach zero for long times as a new equilibrium state is attained. However, as seen in the figure, the 

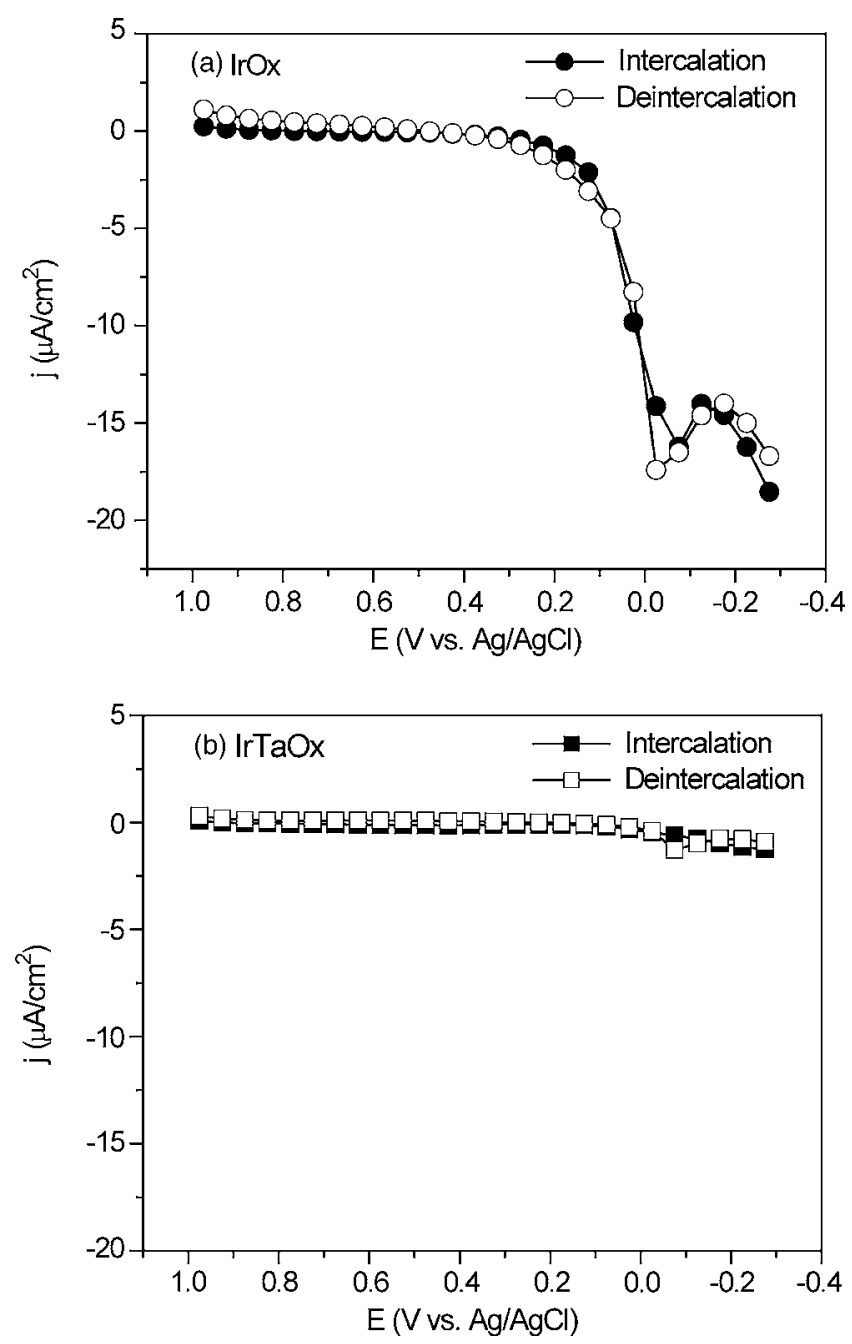

FIG. 2. Steady state current as a function of applied potential for IrOx (a) and $\operatorname{IrTaOx}(\mathrm{b})$, as measured during intercalation and deintercalation.

current attains a steady state. Figure 2 shows the steady state current as a function of potential for (a) IrOx and (b) IrTaOx. It is evident that the steady state current is negative for low potentials and positive at high potentials and that it is an order of magnitude higher for the IrOx sample than for the IrTaOx sample at low potentials. The background current is believed to correspond to oxidation and reduction of different solution species, such as oxygen dissolved in the electrolyte.

In order to proceed with the PITT analysis, an accurate correction for the side reaction current is of importance. If the side reactions operate under charge transfer kinetics, the corresponding current can be described by the Butler-Volmer equation, ${ }^{1}$

$$
j=j_{0}\left[e^{-\alpha n F \eta / R T}-e^{(1-\alpha) n F \eta / R T}\right],
$$

where $\alpha$ is a transfer coefficient between 0 and $1, n$ is the number of electrons per oxidized/reduced molecule, $F$ is Faraday's constant, $R$ is the molar gas constant, $T$ is the temperature, and $\eta$ is the over potential. Provided that the electrode material does not participate in the side reaction and that the catalytic properties of the electrode are independent of potential, Eq. (3) is time independent. For this reason
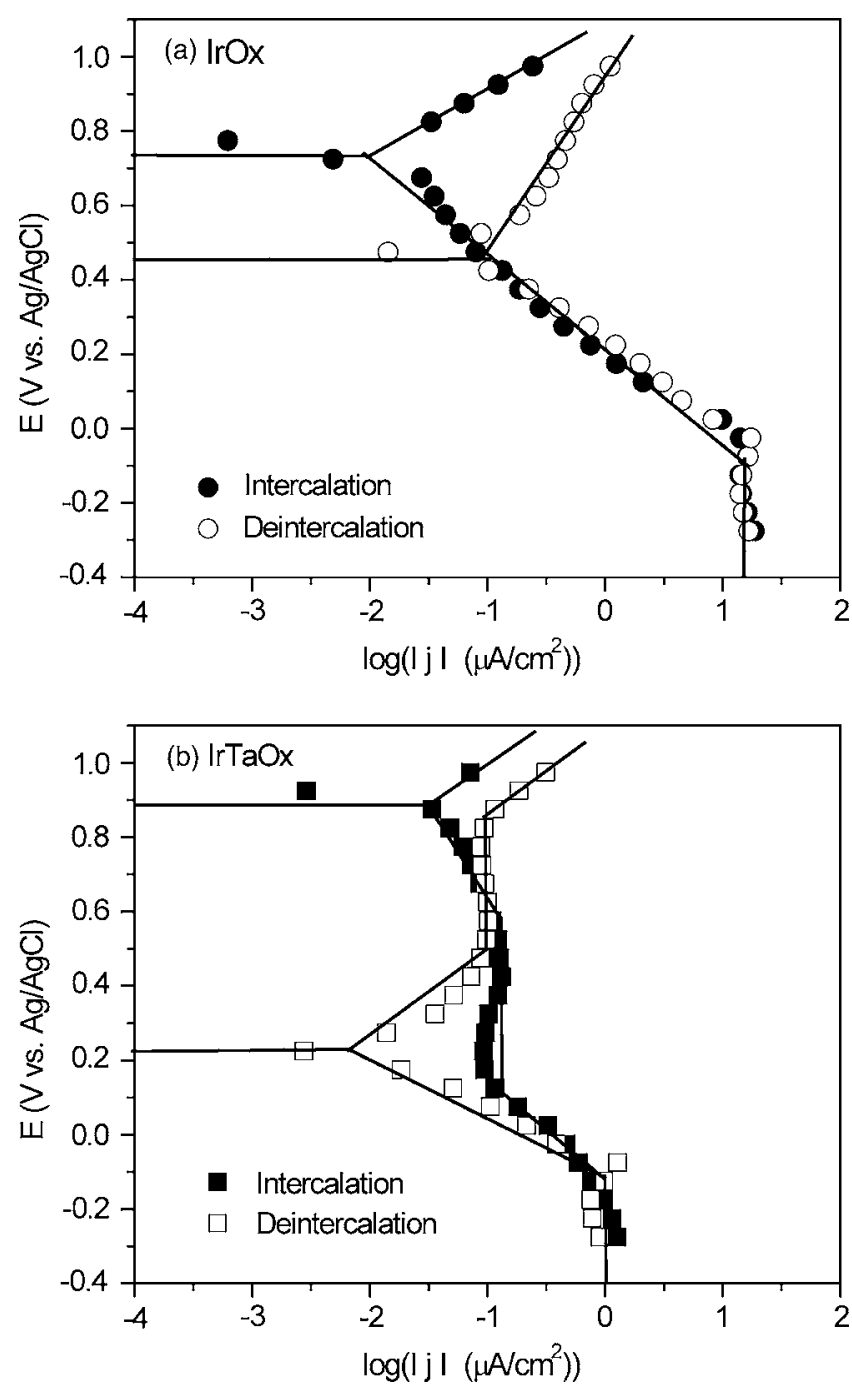

FIG. 3. Evans diagrams, with potential as a function of steady state current density, for $\mathrm{IrOx}(\mathrm{a})$ and $\operatorname{IrTaOx}(\mathrm{b})$.

the side reaction current should be constant during each potential step as long as it is charge transfer limited. This would make the correction easy, as the current associated with the side reactions can be identified as the steady state current. However, the analysis would become much more complicated if the side reactions are solution mass transfer controlled, as the associated current would decrease from an initially high level to a lower steady state current. ${ }^{1}$

To study the kinetics of the side reactions, Evans diagrams, with the potential as a function of the logarithm of the steady state current density, are plotted for IrOx and IrTaOx in Figs. 3(a) and 3(b), respectively. One can find potential regions where the functional dependence is close to linear or horizontal. Within these regions, the side reaction current is charge transfer limited, as given by Eq. (3). The Evans diagrams show different side reaction equilibrium potentials (seen as horizontal lines) for intercalation and deintercalation, indicating that the electrode surface is dependent on the step direction. However, since the potential steps are small and in the same direction during intercalation and during deintercalation, respectively, these effects are assumed to be 
insignificant during each step. The measured current can therefore easily be corrected from the influence of side reactions by subtracting the steady state current.

One can also find potential regions in the Evans diagrams with close to vertical lines. These show the diffusion controlled regimes of the side reaction kinetics, where the diffusion rate of one of the reacting species controls the reaction rate. Since the side reaction current varies during these potential steps, a correct compensation for the side reactions would become very difficult. For simplicity we will consider the background current as constant, also in these regions, and correct the current by subtracting the steady state current.

As a result of analyzing the Evans diagrams it can be concluded that for IrOx, the side reaction currents are constant during each potential step between 1.0 and $0 \mathrm{~V}$ vs $\mathrm{Ag} / \mathrm{AgCl}$, whereas diffusion control is present below $0 \mathrm{~V}$, thus making the analysis less reliable in this region. The situation is much more complex for IrTaOx, which displays charge transfer control in the following regions: $-0.1-0.2$ and above $0.6 \mathrm{~V}$ during intercalation and $-0.1-0.5$ and above $0.8 \mathrm{~V}$ during deintercalation. The other potential regions show signs of mass transfer control. However, the side reaction current is an order of magnitude lower for $\operatorname{IrTaOx}$, making the compensation for side reaction currents less important.

In this study, the constant side reaction current is calculated as the mean current during the last $30 \mathrm{~s}$ of each step. However, the background current is believed to be comparable with, or larger than, the intercalating current for intermediate times. For this reason only Eq. (1) will be used to calculate the diffusion coefficient, since a small error in correcting for side reactions would have a big impact when determining $D$ using Eq. (2).

The expressions for the intercalating current given by Eqs. (1) and (2) are valid provided that the intercalation current is solid phase diffusion limited, which occurs when the diffusion resistance $R_{d}$ is much larger than the interfacial charge transfer resistance $R_{\mathrm{ct}}$ and the sum of Ohmic resistances in the electrolyte, substrate, wires, and connectors, $R_{\text {Ohm }}$. The current response to a potential step, where $R_{\text {Ohm }}$ and $R_{\mathrm{ct}}$ cannot be neglected, was investigated theoretically by Montella. ${ }^{11}$ It was found that if a peak is found in a plot of $I t^{1 / 2}$ versus time instead of an extended plateau towards short times, the diffusion coefficient is underestimated using Eq. (1). The magnitude of the underestimation, given elsewhere, ${ }^{11}$ depends on the dimensionless parameter $\Lambda$, given by

$$
\Lambda=\frac{R_{d}}{R_{\mathrm{Ohm}}+R_{\mathrm{ct}}} .
$$

Figure 4 shows the side reaction compensated $I t^{1 / 2}$ versus the logarithm of time for five different potential steps for (a) IrOx and (b) IrTaOx during the first $100 \mathrm{~s}$ of deintercalation. Instead of extended plateaus where $I t^{1 / 2}$ is time independent, one can only find peaks indicating that $R_{d}$ is small compared to $R_{\text {ct }}$ and $R_{\mathrm{Ohm}}$. Hence only an underestimated diffusion coefficient can be obtained using the peak values found in Fig. 4 together with Eq. (1). This is shown in Fig. 5 for (a) IrOx and (b) IrTaOx during intercalation and deintercalation.
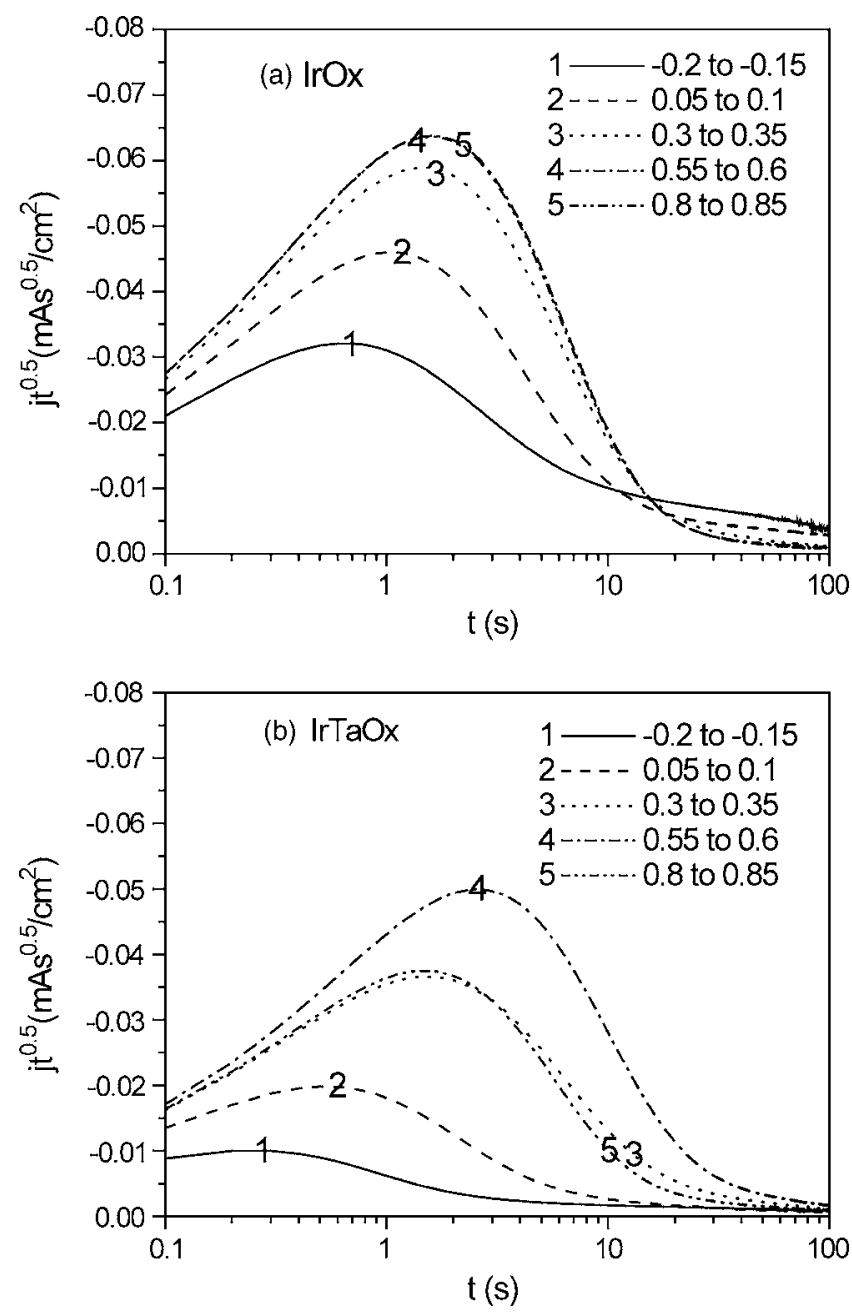

FIG. 4. Time dependence of $j t^{0.5}$ for five different potential steps, as shown in the figure, for $\operatorname{IrOx}(a)$ and $\operatorname{IrTaOx}(b)$.

\section{EIS}

Impedance spectroscopy offers a more accurate determination of the diffusion coefficient, since the interfacial and diffusion processes are usually separated on the frequency scale. ${ }^{10}$ In addition, $\Lambda$ may also be determined. The drawback of EIS is that the data need to be analyzed by fitting a model representative of the system. As a consequence, influences from the side reactions need to be taken into account in the model. For intercalation materials, the EIS data are usually fitted to a Randles circuit, which consists of an Ohmic resistance $R_{\mathrm{Ohm}}$, a charge transfer resistance $R_{\mathrm{ct}}$, a double layer capacitance $C_{\mathrm{dl}}$, and an open Warburg impedance $Z_{W}$ as depicted in Fig. 6. The open Warburg impedance can be represented by a distributed equivalent circuit (transmission line $)^{12,34}$ as depicted in Fig. 7, where $r_{m}$ is the resistance per unit length and $c_{m}$ is the capacitance per unit length. The Randles circuit would, in a complex impedance plot, produce a semicircle, corresponding to charging of the double layer through a charge transfer resistance, followed by a $45^{\circ}$ slope indicating semiinfinite diffusion, which is terminated by a vertical line as a result of the electrode having a finite capacity, as depicted in Fig. 8.

Figure 9 shows two typical impedance spectra for (a) IrOx and (b) IrTaOx, one recorded at high potential and the 

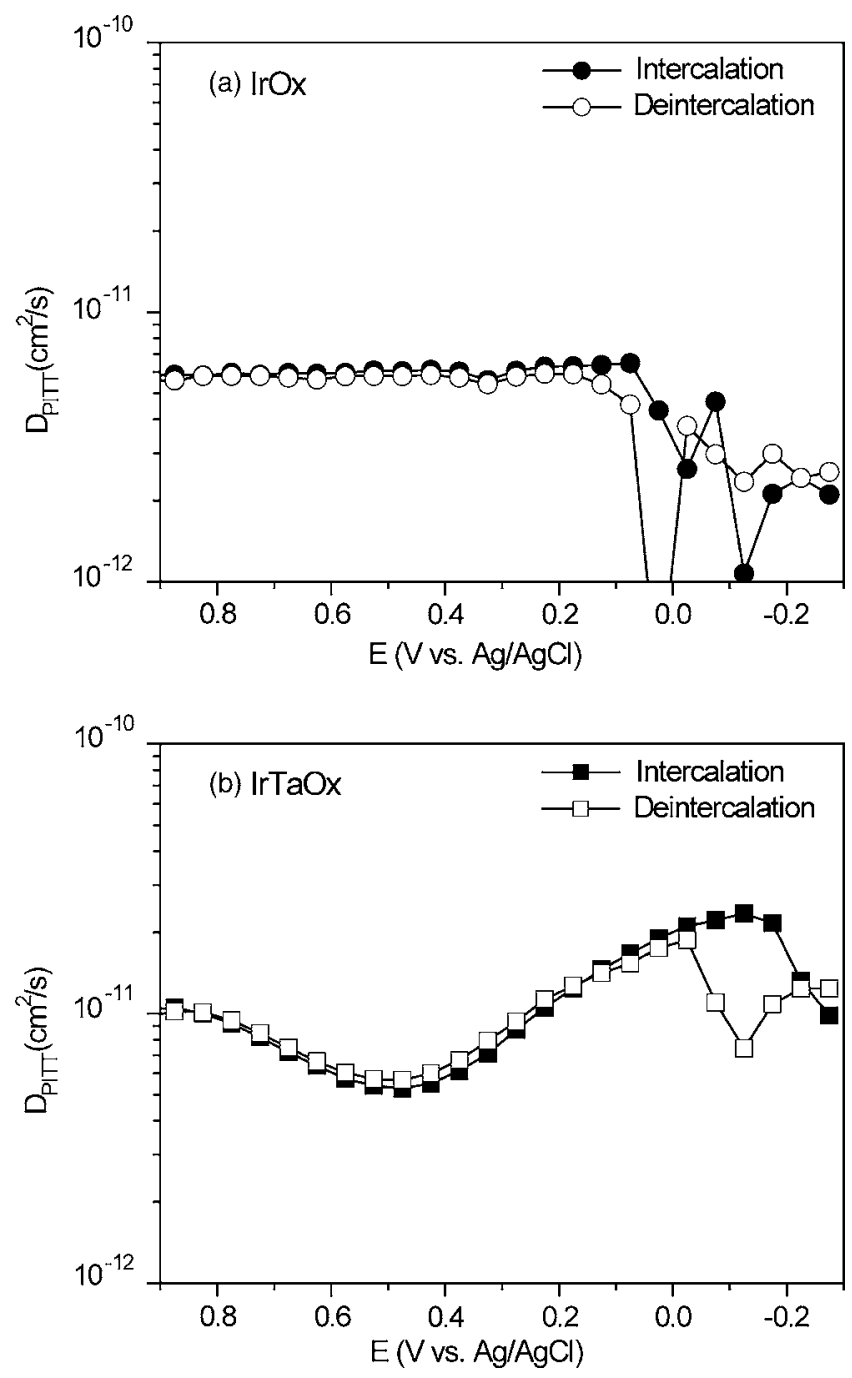

FIG. 5. Diffusion coefficients as a function of potential during intercalation and deintercalation determined using PITT for IrOx (a) and IrTaOx (b).

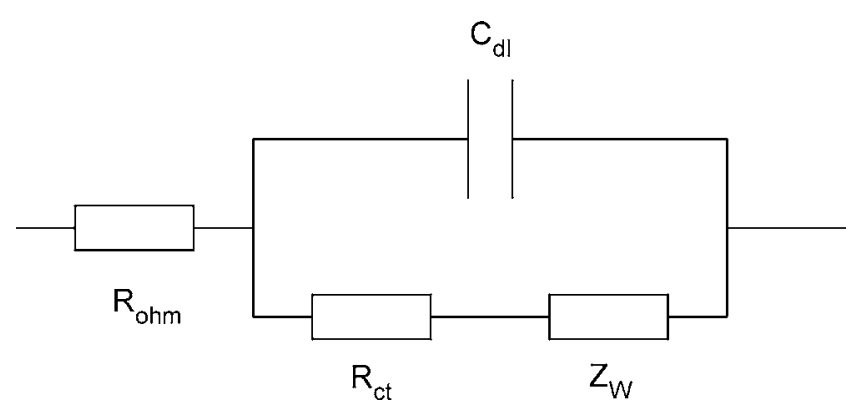

FIG. 6. Randles circuit containing Ohmic resistance $R_{\text {Ohm }}$, double layer capacitance $C_{\mathrm{dl}}$, charge transfer resistance $R_{\mathrm{ct}}$, and a Warburg impedance $Z_{W}$.

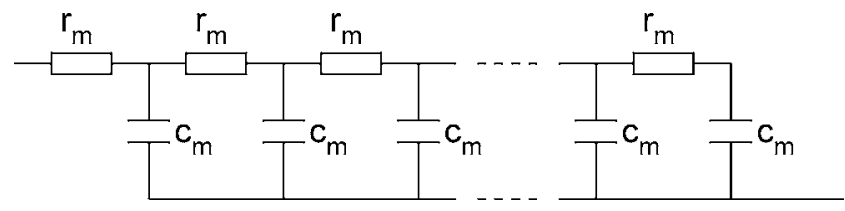

FIG. 7. Transmission line representation of an open Warburg element.

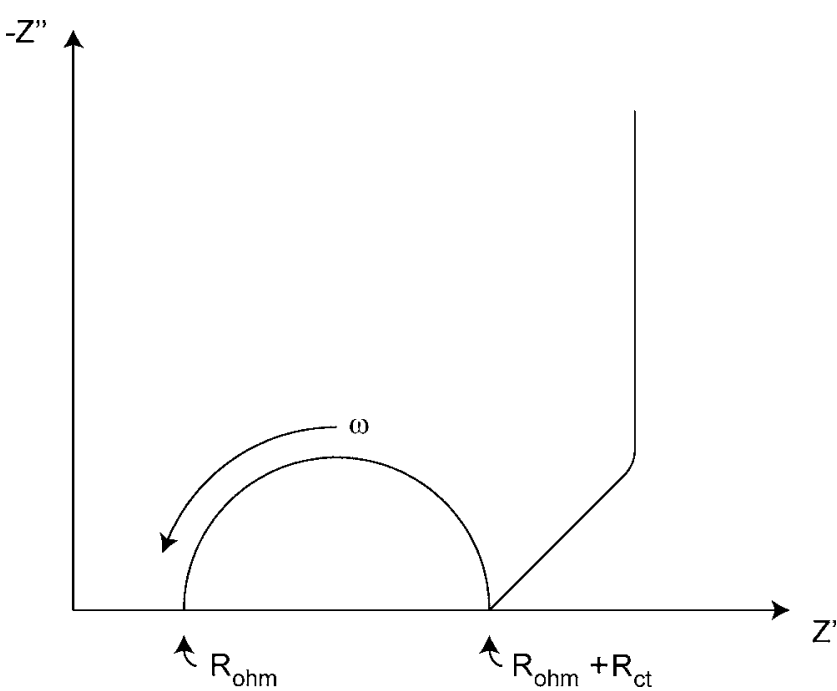

FIG. 8. Impedance of a Randles circuit.
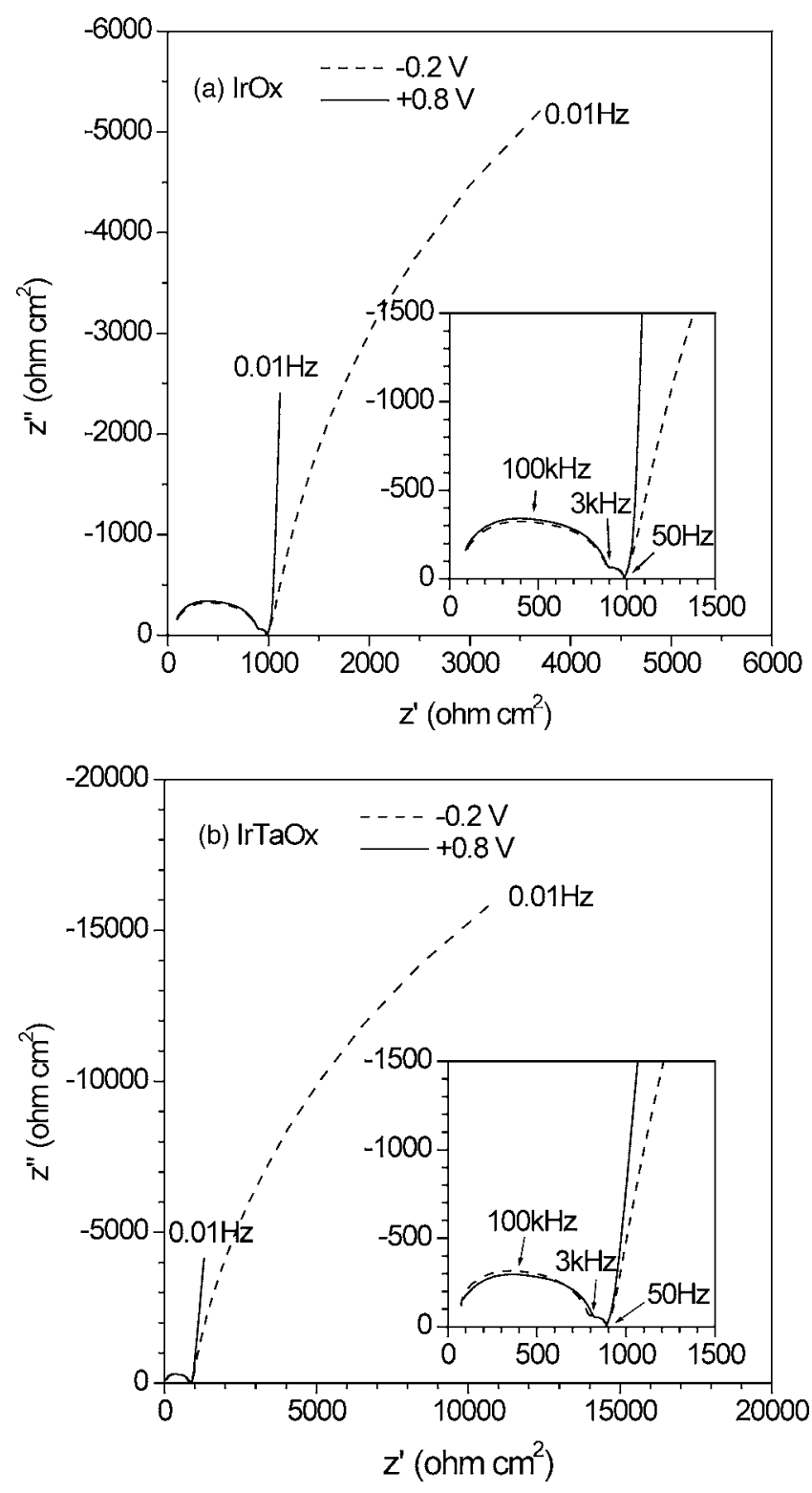

FIG. 9. Complex impedance plots at two potentials for IrOx (a) and IrTaOx (b). The insets show the high frequency region in detail. Some frequencies are indicated on each curve. 


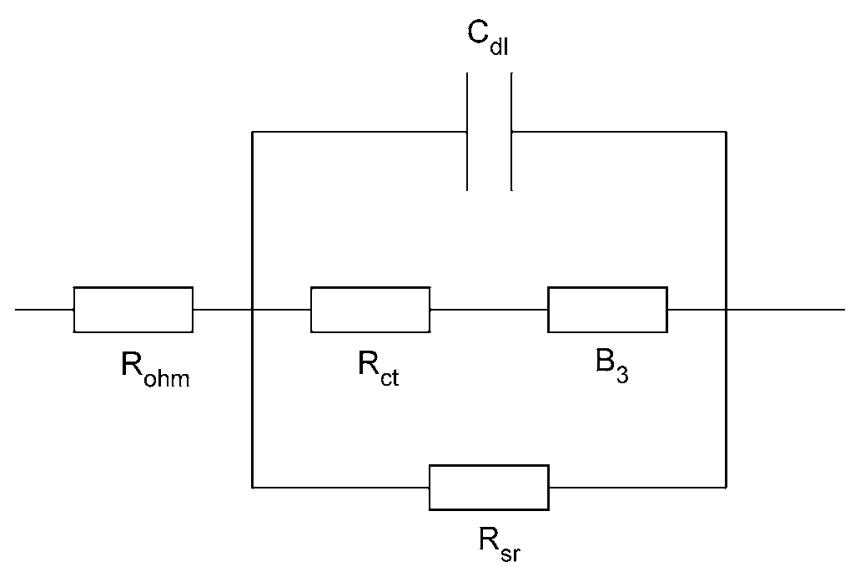

FIG. 10. Equivalent circuit used for fitting experimental impedance spectra, containing Ohmic resistance $R_{\text {Ohm }}$, double layer capacitance $C_{\mathrm{dl}}$, charge transfer resistance $R_{\mathrm{ct}}$, a Bisquert 3 element $B_{3}$, and side reaction resistance $R_{\mathrm{sr}}$.

other at low potential. It can be seen that the response in the high frequency region varies very little for different bias potentials, whereas the low frequency region displays parts of a semicircle for low potentials. This low frequency feature at low potentials is a result of the same side reaction as the one described above in the PITT section. The small semicircle between $50 \mathrm{~Hz}$ and $3 \mathrm{kHz}$ has been identified as the charge transfer resistance and the double layer capacitance, whereas the feature above $3 \mathrm{kHz}$ has been identified as an Ohmic resistance, mainly from the electrolyte, and a frequency dependent capacitive component, which probably is related to the reference electrode.

In order to determine the diffusion coefficient and the $\Lambda$ parameter, several modifications of the Randles circuit were evaluated using the ZVIEW software. ${ }^{35}$ The best fit between model and experiment data in the range of $1 \mathrm{kHz}$ to $0.01 \mathrm{~Hz}$ was achieved by using the equivalent circuit shown in Fig. 10. This model contains an Ohmic resistance $R_{\text {Ohm }}$, a charge transfer resistance $R_{\mathrm{ct}}$, a double layer capacitance $C_{\mathrm{dl}}$, a "Bisquert 3 element" ${ }^{\prime 35} B_{3}$, and a side reaction resistance $R_{\mathrm{sr}}$. This Bisquert element describes an anomalous diffusion process denoted as AD1b by Bisquert and Compte. ${ }^{12}$ The physical realization of such a process is, in our case, most probably percolation or diffusion on a fractal network, leading to the power-law distribution of relaxation times. Addition of the side reaction resistor enabled fitting in the low frequency region, in particular, at low bias potentials.

The model described above resulted in accurate fits of the experimental data. A measure of the accuracy of each fit is the weighted sum of squares, which is proportional to the average percentage error between the experimental data points and the simulated values. It was typically around 0.004 never exceeding 0.04 . Figure 11 shows the experimental data of $\mathrm{IrTaOx}$ at $0 \mathrm{~V}$ vs $\mathrm{Ag} / \mathrm{AgCl}$ together with its corresponding fit. This fit is one of the poorest, with a weighted sum of squares of 0.01 .

The solid phase diffusion coefficient was determined from the fitted parameters of the $B_{3}$ element in the circuit. This element is most easily visualized by a transmission line,

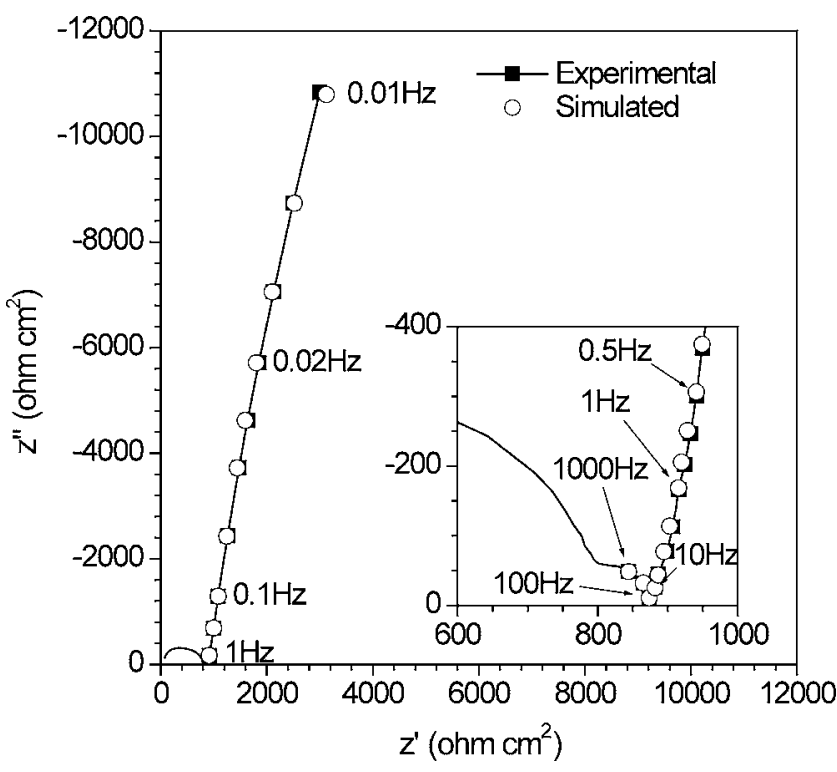

FIG. 11. Complex impedance plots of experimental and fitted data for $\mathrm{IrTaOx}$ at $0 \mathrm{~V}$ vs $\mathrm{Ag} / \mathrm{AgCl}$. The insets show the high frequency region in detail. Some frequencies are indicated on each curve.

illustrated in Fig. 12, where the distributed resistor for the ordinary diffusion has been replaced by a distributed constant phase element $(\mathrm{CPE})$ with impedance

$$
\chi_{m}(j \omega)=q_{m}(j \omega)^{-(1-\gamma)},
$$

where $1-\gamma$ is the distributed $\mathrm{CPE}$ exponent, which is equal to zero for ordinary diffusion, and $q_{m}$ is the distributed CPE prefactor, which has the dimension $(\Omega / m) s^{-(1-\gamma)}$. The impedance for the $B_{3}$ element is equal to ${ }^{12}$

$$
Z(j \omega)=R_{W} \omega_{d}^{\gamma-1}\left(\frac{\omega_{d}}{j \omega}\right)^{1-\gamma / 2} \operatorname{coth}\left[\left(\frac{j \omega}{\omega_{d}}\right)^{\gamma / 2}\right],
$$

where $R_{W}$ is given by

$$
R_{W}=q_{m} L
$$

the characteristic angular frequency $\omega_{d}$ by

$$
\omega_{d}=\left(\frac{D}{L^{2}}\right)^{1 / \gamma}
$$

and $D$ is given as ${ }^{12}$

$$
D=\frac{1}{c_{m} q_{m}} .
$$

It should be noted that $D$ and $R_{W}$, in Eqs. (9) and (7), have the dimensions $\mathrm{m}^{2} / \mathrm{s}^{\gamma}$ and $\Omega s^{-(1-\gamma)}$, respectively, which makes it impossible to use $R_{W}$ as a diffusion resistance for

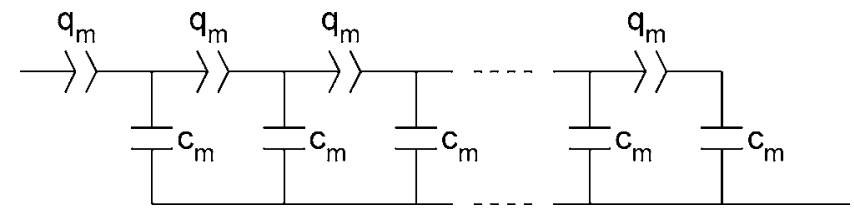

FIG. 12. Transmission line representation of the Bisquert element $B_{3}$ describing anomalous diffusion. 

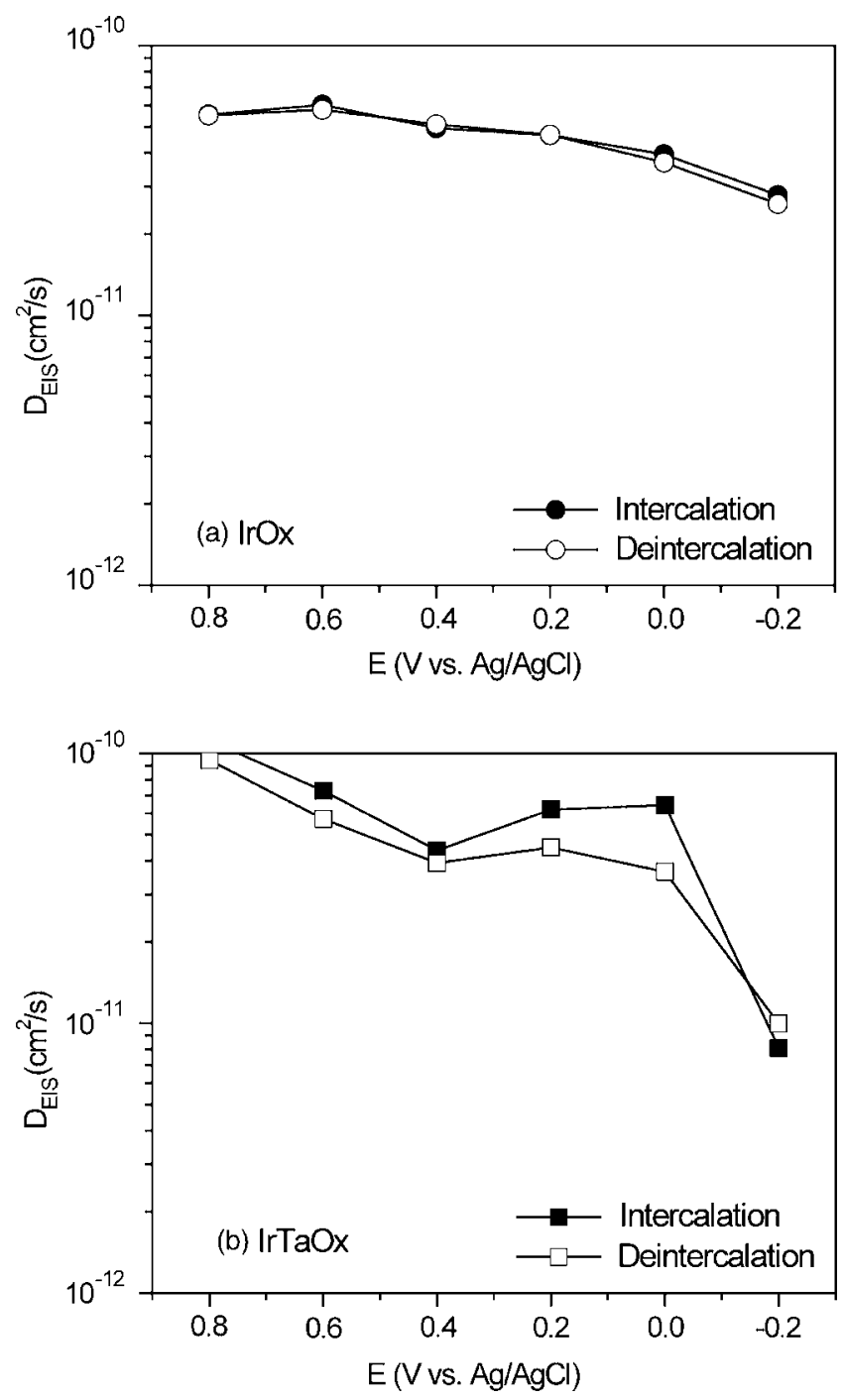

FIG. 13. Diffusion coefficients as a function of potential determined using EIS, after each intercalation and deintercalation step, for IrOx (a) and $\operatorname{IrTaOx}(b)$.

calculating the $\Lambda$ parameter. However, an effective $R_{d}$ can be identified from Eq. (6) as

$$
R_{d, \mathrm{eff}}=R_{W} \omega_{d}^{-(1-\gamma)}
$$

which can be used to calculate $\Lambda$ from Eq. (4). This further implies that an effective diffusion coefficient with dimension $\mathrm{m}^{2} / \mathrm{s}$ can be calculated as

$$
D_{\text {eff }}=D \omega_{d}^{1-\gamma} \text {. }
$$

Figure 13 shows the values of the effective diffusion coefficient for (a) IrOx and (b) IrTaOx measured using EIS. Comparing these results with diffusion coefficient measured using PITT (Fig. 5), it can be seen that the diffusion coefficient measured using EIS is up to a factor of 10 larger than the one measured using PITT. Figure 14 shows the Warburg exponent identified as $(1-\gamma / 2)$ and the $\Lambda$ parameter for (a) IrOx and (b) IrTaOx measured using EIS. One can see that $\Lambda$ is low $(<1)$ in the high potential range, and that the Warburg exponent increases from 0.65 to 0.75 for $\mathrm{IrOx}$ and 0.7 to 0.85 for $\mathrm{IrTaOx}$ when the potential is decreased from 0.8 to $-0.2 \mathrm{~V}$ vs $\mathrm{Ag} / \mathrm{AgCl}$.
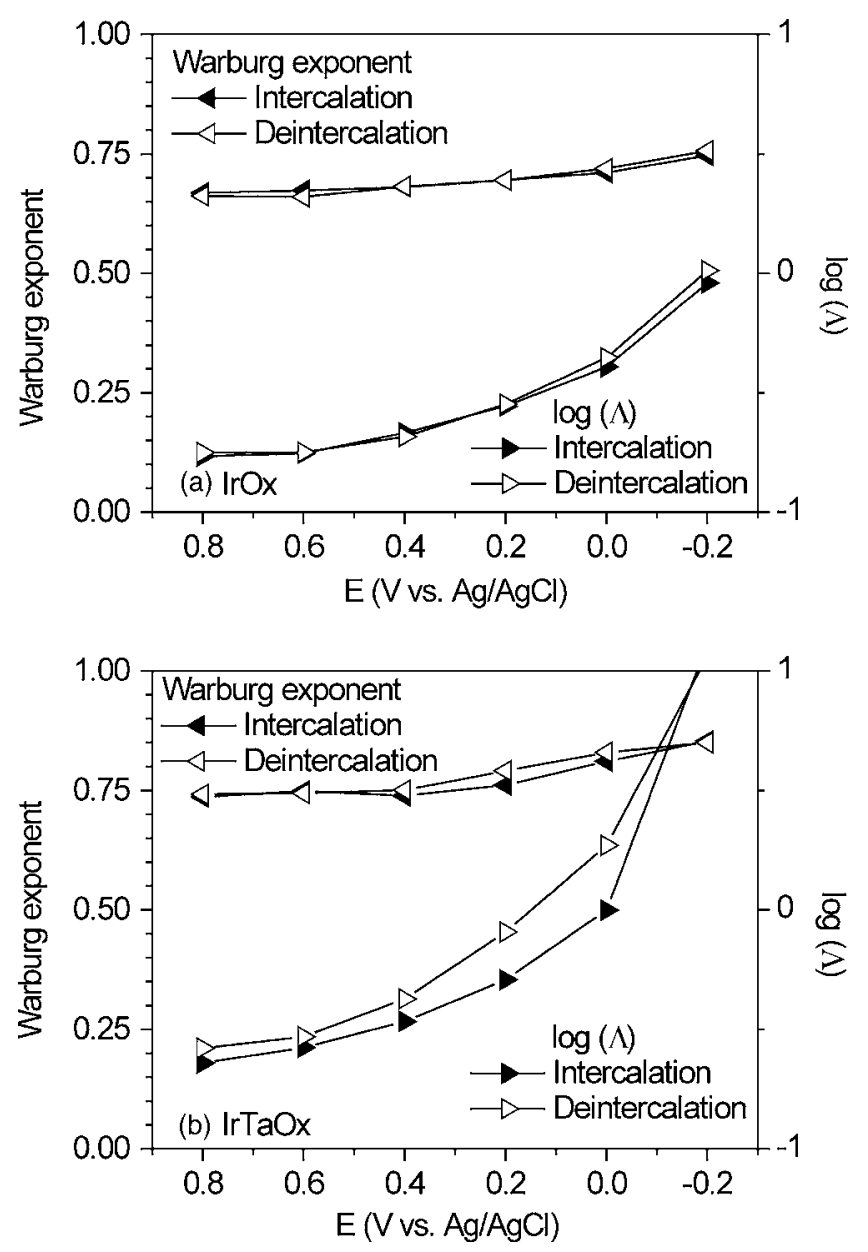

FIG. 14. Warburg exponent and $\Lambda$ parameter as a function of potential, as determined by EIS, after each intercalation and deintercalation step, for IrOx (a) and $\operatorname{IrTaOx}(b)$.

\section{RESULTS AND ANALYSIS: DENSITY OF STATES PITT}

It has previously been reported that it is possible to probe the electronic density of states using GITT or CP (Ref. 14) since it can be expressed as

$$
\mathrm{DOS}=-\frac{1}{e} \frac{d x}{d U}
$$

where $e$ is the elementary charge, $x$ is the number of intercalated ion-electron pairs per f.u., and $d U$ is the change of potential. A condition for this relation is that the rigid band approximation can be applied to the oxides, which means that the DOS of the oxide is not affected by the intercalated ions. Another possible condition is that the sites for the inserted electrons are localized. ${ }^{14}$

When using PITT, $d U$ is the applied potential step and $d x$ can be calculated from

$$
d x=\frac{\int I(t) d t / e}{N_{\mathrm{Ir}}},
$$

where $I(t)$ is the current as a function of $t$ and $N_{\text {Ir }}$ is the number of iridium atoms determined by Rutherford backscattering spectroscopy. 

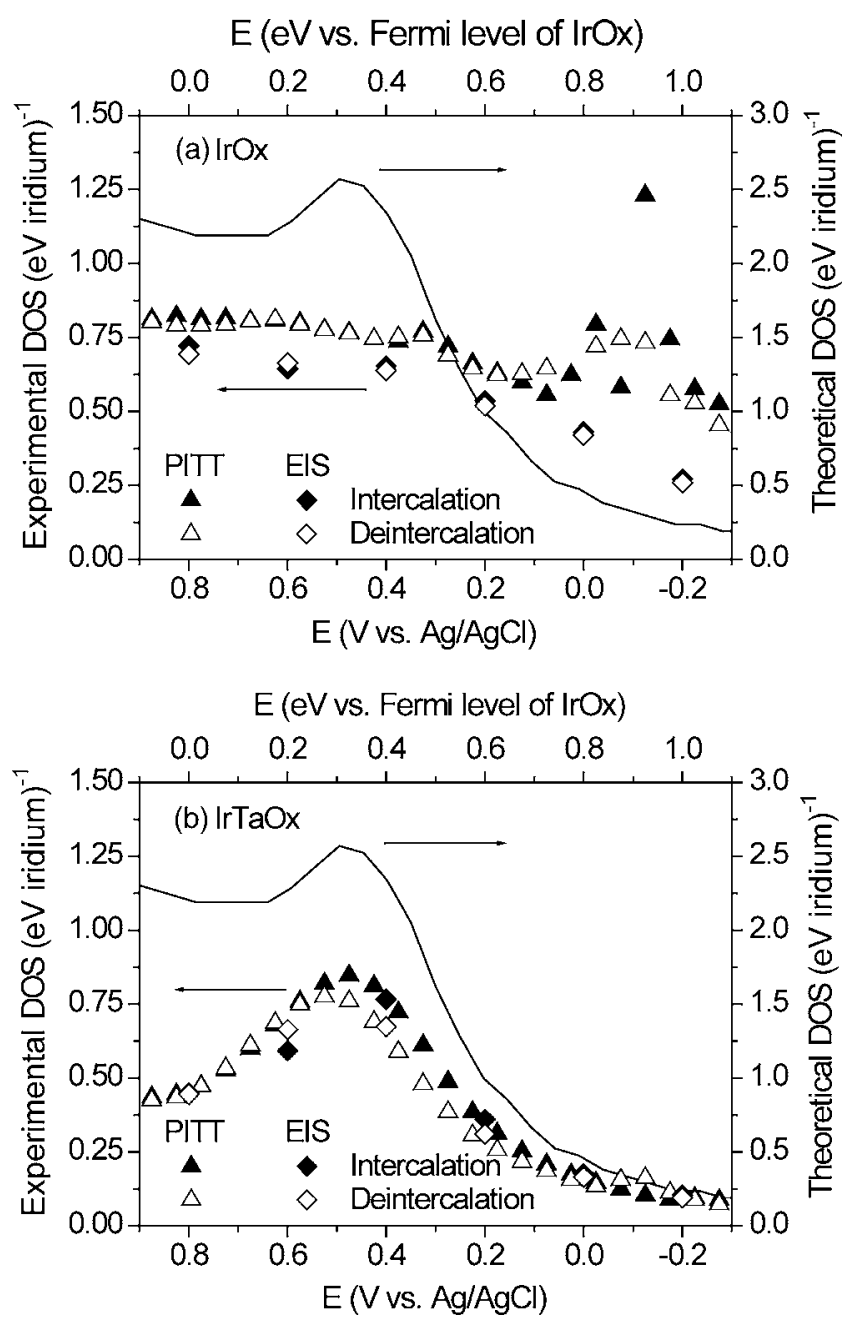

FIG. 15. Experimental DOS determined by PITT and EIS, during intercalation and deintercalation, for $\operatorname{IrOx}$ (a) and $\operatorname{IrTaOx}$ (b) together with the theoretical DOS of iridium oxide calculated by Xu et al. (Ref. 36).

\section{EIS}

For determining the DOS, impedance spectroscopy was used to measure the capacitance in the low frequency region. As the capacitance describes how much an ac potential changes the intercalated charge, it becomes evident that the measured low frequency capacitance $C$ is proportional to the DOS according to

$$
\mathrm{DOS}=\frac{C / e^{2}}{N_{\mathrm{Ir}}},
$$

where $C$ is calculated from

$$
C=c_{m} L \text {. }
$$

Figure 15 shows the calculated DOS for (a) $\mathrm{IrOx}$ and (b) IrTaOx together with a theoretical DOS of crystalline $\mathrm{IrO}_{2}{ }^{36}$ It can be seen that there are only small differences between the DOS measured with PITT and EIS. However, the measured DOS is $30 \%-50 \%$ of the theoretical DOS.

\section{DISCUSSION}

Investigating the chemical diffusion coefficient using PITT requires knowledge about the kinetics of the intercala- tion process. The $\Lambda$ parameter, which is the ratio of diffusion resistance to the sum of charge transfer resistance and the Ohmic resistances, gives an indication whether the current is limited by a diffusion process or by an interfacial process and the electrolyte. Montella ${ }^{11}$ presented a method to calculate the chemical diffusion coefficient using PITT for systems where the charge transfer and Ohmic resistances could not be neglected, and to determine the $\Lambda$ parameter. However, the applicability of this method is uncertain for our films since a significant side reaction has been identified. EIS on the other hand offers an easy way in determining both diffusion coefficient and $\Lambda$ parameter, which follows from the interfacial and diffusion processes being separated on the frequency scale. In addition the side reaction can easily be identified. The diffusion coefficient was determined to be in the $10^{-11}-10^{-10} \mathrm{~cm}^{2} / \mathrm{s}$ range for both $\operatorname{IrOx}$ and $\operatorname{IrTaOx}$, which is two orders of magnitude larger than previously reported by us for similar films, ${ }^{32}$ but three orders of magnitude smaller than reported by Pauporté and Durand. ${ }^{37}$

The diffusion coefficient determined by PITT is smaller than the diffusion coefficient determined by EIS, most probable as a result of small $\Lambda$ values. To strengthen this latter statement, the ratio between the diffusion coefficients measured by PITT and EIS was plotted as a function of the $\Lambda$ parameter together with the theoretical ratio calculated by Montella ${ }^{11}$ in Fig. 16(a) for IrOx and Fig. 16(b) for IrTaOx. A good agreement between theory and experiment can be seen for high potentials. Discrepancies at low potentials are ascribed to the effect of the questionable side reaction compensation. It is, however, important to notice that EIS showed that the diffusion is anomalous with a Warburg exponent $(1-\gamma / 2)$ ranging from $0.65-0.75$ for $\mathrm{IrOx}$ and $0.7-$ 0.85 for IrTaOx, as compared to 0.5 for normal diffusion, whereas the analysis of PITT data did not consider anomalous diffusion. The measured Warburg exponent is close to the value 0.73 theoretically predicted for percolation conductivity, ${ }^{38}$ and to the exponents observed in a number of ion conducting glasses. ${ }^{39}$

The determination of DOS showed good agreement between PITT and EIS, and it can be seen that the measured DOS shows the same main features as the theoretical DOS with a peak slightly above the Fermi level and a declining DOS for higher energies. This agreement is particularly good for IrTaOx, whereas the measured DOS of IrOx show less features, which is interpreted as a result of the $\mathrm{IrOx}$ being more affected by the side reactions. The measured DOS does, however, show a lower amplitude than the theoretical one in the entire potential range, which is in agreement with previous work on $\mathrm{TiO}_{2}$ and $\mathrm{WO}_{3}$. ${ }^{14}$

Previous work has shown that GITT and chronopotentiometry can be used to probe the DOS. We are now suggesting that also PITT and EIS are capable to probe the DOS, and are even more suitable for doing so. The biggest advantage of PITT and EIS compared to GITT or CP is the ability to detect, and to some extent compensate, for the side reactions occurring at the sample. From the background current one can draw the conclusion that the results are more reliable for high potentials where the background current is small and charge transfer kinetic controlled, and that the uncertainty is 

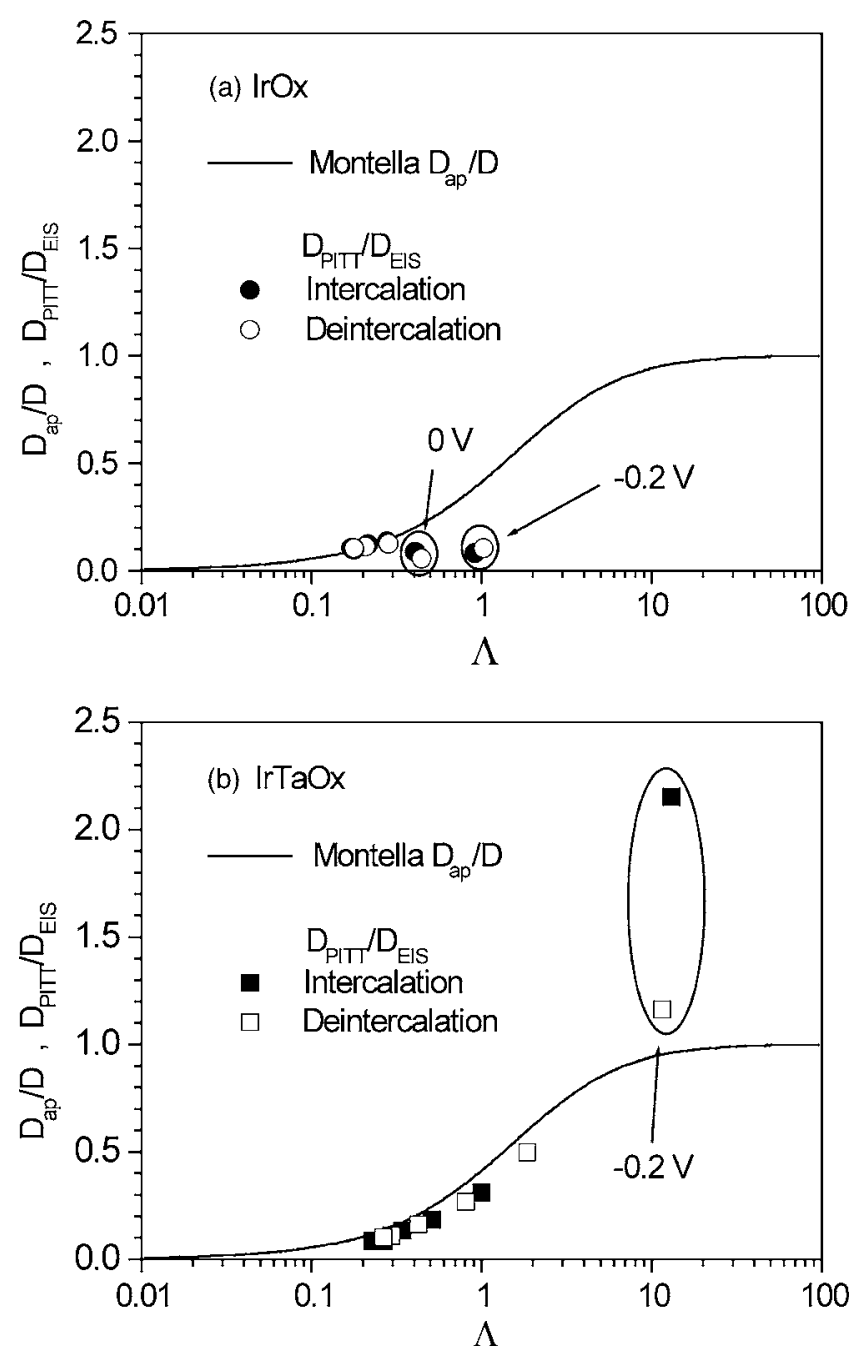

FIG. 16. Ratio between the diffusion coefficients measured by PITT and EIS, during intercalation and deintercalation, for IrOx (a) and $\operatorname{IrTaOx}(b)$ together with the theoretical ratio calculated by Montella (Ref. 11).

largest for IrOx at low potentials where the background current is mass transfer limited. It is also realized that the intercalated charge increases for longer times than those used in these experiments. However, most of the charge is intercalated in the beginning of the potential step and the influence of side reactions increases with time.

\section{CONCLUSIONS}

Thin film samples of $\mathrm{IrOx}$ and $\mathrm{IrTaOx}$ were prepared by reactive dc magnetron sputtering. PITT and EIS were used to determine the chemical diffusion coefficient and the DOS, and a significant side reaction could be observed and corrected for. It was shown that the traditional PITT approach underestimates the chemical diffusion coefficient since the charge transfer and Ohmic resistances cannot be neglected when analyzing PITT data, whereas EIS can be used to obtain good estimates of the chemical diffusion coefficient as well as diffusion, charge transfer, and Ohmic resistances. The diffusion coefficient was determined to be in the $10^{-11}-10^{-10} \mathrm{~cm}^{2} / \mathrm{s}$ range for both $\mathrm{IrOx}$ and $\mathrm{IrTaOx}$, and the measured DOS shows features of the theoretically calculated DOS of crystalline iridium oxide, the agreement is particularly good for IrTaOx.

\section{ACKNOWLEDGMENTS}

The authors are thankful for financial support from the Swedish Research Council and valuable discussions with Professor C. G. Granqvist.

${ }^{1}$ J. B. Bard and L. R. Faulkner, Electrochemical Methods (Wiley, New York, 1980).

${ }^{2}$ Solid State Electrochemistry, edited by P. G. Bruce, B. Dunn, and J. W. Goodby (Cambridge University Press, Cambridge, 1995).

${ }^{3}$ Advances in Lithium-Ion Batteries, edited by B. Scrosati and W. A. van Schalkwijk (Kluwer, New York, 2002).

${ }^{4}$ G. Alberti and M. Casciola, Solid State Ionics 145, 3 (2001).

${ }^{5}$ G. A. Niklasson and C. G. Granqvist, J. Mater. Chem. 17, 127 (2007).

${ }^{6}$ J. Jamnik and J. Maier, Phys. Chem. Chem. Phys. 3, 1668 (2001).

${ }^{7}$ J. Jamnik, Solid State Ionics 157, 19 (2003).

${ }^{8}$ W. Weppner and R. A. Huggins, J. Electrochem. Soc. 124, 1569 (1977).

${ }^{9}$ C. J. Wen, B. A. Boukamp, R. A. Huggins, and W. Weppner, J. Electrochem. Soc. 126, 2258 (1979).

${ }^{10}$ C. Ho, I. D. Raistrick, and R. A. Huggins, J. Electrochem. Soc. 127, 343 (1980).

${ }^{11}$ C. Montella, J. Electroanal. Chem. 518, 61 (2002).

${ }^{12}$ J. Bisquert and A. Compte, J. Electroanal. Chem. 499, 112 (2001).

${ }^{13}$ M. Strømme, R. Ahuja, and G. A. Niklasson, Phys. Rev. Lett. 93, 206403 (2004).

${ }^{14}$ G. A. Niklasson, R. Ahuja, and M. Strømme, Mod. Phys. Lett. B 20, 863 (2006).

${ }^{15}$ G. A. Niklasson, L. Berggren, A. K. Jonsson, R. Ahuja, N. V. Skorodumova, J. Backholm, and M. Strømme., Sol. Energy Mater. Sol. Cells 90, 385 (2006).

${ }^{16}$ I. Pollini, A. Mosser, and J. C. Parlebas, Phys. Rep. 355, 1 (2001).

${ }^{17}$ J. J. Rehr and A. L. Ankudinov, Coord. Chem. Rev. 249, 131 (2005).

${ }^{18}$ J. R. Dahn, J. N. Reimers, T. Tiedje, Y. Gao, A. K. Sleigh, W. R. McKinnon, and S. Cramm, Phys. Rev. Lett. 68, 835 (1992).

${ }^{19}$ T. Saito, T. Furuta, J.-H. Hwang, S. Kuramoto, K. Nishino, N. Suzuki, R. Chen, A. Yamada, K. Ito, Y. Seno, T. Nonaka, H. Ikehata, N. Nagasako, C. Iwamoto, Y. Ikuhara, and T. Sakuma, Science 300, 464 (2003).

${ }^{20} \mathrm{H}$. W. Hugosson, U. Jansson, B. Johansson, and O. Eriksson, Science 293, 2434 (2001).

${ }^{21}$ L. S. Dubrovinsky, N. A. Dubrovinskaia, V. Swamy, J. Muscat, N. M. Harrison, R. Ahuja, B. Holm, and B. Johansson, Nature (London) 410, 653 (2001).

${ }^{22}$ P. Sharma, A. Gupta, K. V. Rao, F. J. Owens, R. Sharma, R. Ahuja, J. M. Osorio Guillen, B. Johansson, and G. A. Gehring, Nat. Mater. 2, 673 (2003).

${ }^{23}$ R. S. Crandall and B. W. Faughnan, Phys. Rev. Lett. 39, 232 (1977).

${ }^{24}$ C. G. Granqvist, Handbook of Inorganic Electrochromic Materials (Elsevier, Amsterdam, 1995).

${ }^{25}$ G. Beni and J. L. Shay, Appl. Phys. Lett. 33, 567 (1978).

${ }^{26}$ J. L. Shay, G. Beni, and L. M. Schiavone, Appl. Phys. Lett. 33, 942 (1978).

${ }^{27}$ S. Gottesfeld, J. D. E. McIntyre, G. Beni, and J. L. Shay, Appl. Phys. Lett. 33, 208 (1978).

${ }^{28}$ L. M. Schiavone, W. C. Dautremont-Smith, G. Beni, and J. L. Shay, Appl. Phys. Lett. 35, 823 (1979).

${ }^{29}$ E. Avendano, A. Azens, G. A. Niklasson, and C. G. Granqvist, Sol. Energy Mater. Sol. Cells 84, 337 (2004).

${ }^{30}$ T. Niwa, K. Uchikawa, and T. Endo, Japan Display 1986 (The Society for information and display and The Institute of Television Engineers of Japan, Japan, 1986), pp. 372-375.

${ }^{31}$ A. Azens and C. G. Granqvist, Appl. Phys. Lett. 81, 928 (2002).

${ }^{32} \mathrm{~J}$. Backholm, A. Azens, and G. A. Niklasson, Sol. Energy Mater. Sol. Cells 90, 414 (2006).

${ }^{33}$ J. Backholm, E. Avendano, A. Azens, G. de M. Azevedo, E. Coronel, G. A. Niklasson, and C. G. Granqvist, Sol. Energy Mater. Sol. Cells 92, 91 (2008). 
${ }^{34}$ Impedance Spectroscopy, edited by E. Barsoukov and J. R. Mcdonald (Wiley, New Jersey, 2005).

${ }^{35}$ Z VIEW 2.9b, Schribner Associates, Inc., 1990-2005.

${ }^{36}$ J. H. Xu, T. Jarlborg, and A. J. Freeman, Phys. Rev. B 40, 7939 (1989).
${ }^{37}$ Th. Pauporté and R. Durand, J. Appl. Electrochem. 30, 35 (2000).

${ }^{38}$ M. Sahimi, Applications of Percolation Theory (Taylor \& Francis, London 1994).

${ }^{39}$ D. L. Sidebottom, Phys. Rev. Lett. 83, 983 (1999). 